\title{
Semiblind frequency-domain timing synchronization and channel estimation for OFDM systems
}

\author{
Te-Lung Kung* and Keshab K Parhi
}

\begin{abstract}
In this article, we propose unit vectors in the high dimensional Cartesian coordinate system as the preamble, and then propose a semiblind timing synchronization and channel estimation scheme for orthogonal frequency division multiplexing (OFDM) systems. Due to the lack of useful information in the time-domain, a frequency-domain timing synchronization algorithm is proposed. The proposed semiblind approach consists of three stages. In the first stage, a coarse timing offset related to the delayed timing of the path with the maximum gain in multipath fading channels is obtained. Then, a fine time adjustment algorithm is performed to find the actual delayed timing in channels. Finally, the channel response in the frequency-domain is obtained based on the final timing estimate. Although the required number of additions in the proposed algorithm is higher than those in conventional methods, the simulation results show that the proposed approach has excellent performance of timing synchronization in several channel models at signal-to-noise ratio (SNR) smaller than $6 \mathrm{~dB}$. In addition, for a low-density parity-check coded single-input single-output OFDM system, our proposed approach has better bit-error-rate performance than conventional approaches for SNR varying from 3 to $8 \mathrm{~dB}$.
\end{abstract}

Keywords: Timing synchronization, Fine time adjustment, Channel estimation, Orthogonal frequency division multiplexing (OFDM), Unit vectors, Frequency-domain processing

\section{Introduction}

Orthogonal frequency division multiplexing (OFDM) is a promising technology to support high-rate wired and wireless applications due to its robustness to multipath delay spread [1-3]. However, in OFDM systems, synchronization errors can destroy the orthogonality among the subcarriers and result in performance degradation. Thus, timing synchronization in OFDM systems becomes much more challenging due to the increase in the amount of inter-carrier interference (ICI) and inter-symbol interference (ISI) [1]. Although the soft decoders employing error correction code can improve the system performance at low signal-to-noise ratio (SNR), perfect timing synchronization is necessary for the decoder to operate correctly. Therefore, in order to improve the system performance,

*Correspondence: kungx012@umn.edu

Department of Electrical and Computer Engineering, University of Minnesota, Minneapolis, MN, 55455, USA it is important to find the actual delayed timing in multipath fading channels at the receiver. In addition, channel estimation also plays a crucial role in providing the channel information to the soft decoder and compensating the signal during the demodulation process [4]. Without the knowledge of timing offset and channel information at the receiver, the system will have a poor performance during the entire data transmission.

Various synchronization techniques for orthogonal frequency division multiplexing (OFDM) systems have been developed using well-designed preambles [5-14]. Although accurate timing estimation can be achieved, the bandwidth efficiency is also inevitably reduced. In order to reduce the waste of bandwidth, non-data aided synchronization algorithms based on the cyclic prefix (CP) have been proposed $[15,16]$. However, in some multipath fading channels with non-line-of-sight (NLOS) propagation, both data-aided and non-data-aided synchronization methods frequently lead to the delayed timing in chan-

\section{是 Springer}

(c) 2013 Kung and Parhi; licensee Springer. This is an Open Access article distributed under the terms of the Creative Commons Attribution License (http://creativecommons.org/licenses/by/2.0), which permits unrestricted use, distribution, and reproduction in any medium, provided the original work is properly cited. 
nels where the delayed path has larger gain than the first path. In this case, the resulting ICI and ISI would degrade the system performance. Also, the channel coding would not perform well because of the synchronization errors. Therefore, in order to solve this problem, a fine time adjustment is needed to modify the frequently delayed timing to the actual delayed timing in channels. In [15], the proposed timing estimator performs well only for the additive white Gaussian noise (AWGN) channels. While the system operates in the multipath fading channels, the proposed algorithm exhibits significantly large fluctuation in the estimated timing offset. In [16], the modified blind timing synchronization method has a good performance in the multipath fading channels with lineof-sight (LOS) propagation only when the SNR is greater than $20 \mathrm{~dB}$. In [14], a well-designed time-domain training sequence is utilized to perform joint timing synchronization and channel estimation. Although the proposed timing estimator has excellent performance at low SNR [14], the power consumption of the proposed preamble is still too large to be adopted in some low-power wireless applications.

For wireless implantable medical devices, low-power consumption is necessary in order to prolong the battery operating time. This article develops a semiblind timing synchronization and channel estimation algorithm based on unit vectors, and demonstrates that this algorithm is suitable for multipath fading channels with both LOS and NLOS propagation. Due to the use of unit vectors as the preamble, the power consumption of this preamble at the transmitter is approximately equal to zero. Therefore, the proposed preamble is suitable for any low-power wireless implantable medical device. In addition, we utilize only one nonzero sample in the training sequence to perform the timing synchronization, and this training sequence definitely lacks useful information at the receiver. Compared with the existing preamble-based methods [5-9,11,12,14], the number of nonzero elements in the proposed training sequence is the lowest. Thus, the proposed joint approach is called a semiblind method. In this article, we first obtain a coarse timing offset using the cross-correlation function outputs in the frequencydomain. Then, a fine time adjustment algorithm based on these outputs is applied. Finally, the channel response in the frequency-domain is obtained. Simulation results are represented to verify the effectiveness of our proposed algorithm. This article is an extended version of [13].

This article is organized as follows. Section 2 describes the system and the problem. In Section 3, the proposed semiblind timing synchronization and channel estimation algorithm is presented. Simulation results are provided in Section 4, respectively. Finally, Section 5 concludes this article.

\section{Problem statement}

\subsection{System description}

In this article, we consider a training-sequence-based single-input single-output OFDM system as shown in Figure 1. The training sequence is an unit vector in an $N$ dimensional Cartesian coordinate system, where $N$ represents the number of subcarriers in the OFDM system. Let $\mathbf{p}^{T}=\left[\begin{array}{lllllll}0 & \ldots & 0 & 1 & 0 & \ldots & 0\end{array}\right]=\left\{p(n), \forall n \in \Omega_{1}\right\}$ denotes the proposed training sequence, where $\Omega_{1}=\{0,1, \ldots, N-1\}$, $p(n)=\delta(n-c), c \in\{0,1, \ldots, N-1\}$, the length of $\mathbf{p}^{T}$ is $N$, and the power of $\mathbf{p}^{T}$ is equal to $1 / N$. Consider the transmitted packet $\mathbf{s}^{T}=\left[\mathbf{p}^{T} \mathbf{x}^{T}\right]=\left\{s(n), \forall n \in \Omega_{2}\right\}$, where $\mathbf{x}^{T}$ consists of $\ell$ OFDM symbols, the length of $\mathbf{x}^{T}$ is $\ell \cdot\left(N+N_{C P}\right), N_{C P}$ denotes the length of cyclic prefix, $\ell$ is a positive integer, and $\Omega_{2}=\left\{0,1, \ldots, \ell \cdot\left(N+N_{C P}\right)+N-1\right\}$. Assume that the cyclic prefix in each OFDM symbol is longer than the maximum delay spread of the channel, and the path delays in the channels are sample-spaced. Therefore, the received signal at the receiver can be expressed as

$$
r(n)=e^{\frac{j 2 \pi \epsilon n}{N}} \sum_{k=0}^{K-1} h(k) s(n-\tau-k)+w(n),
$$

where $\epsilon$ is the carrier frequency offset (CFO) normalized to the OFDM subcarrier spacing, $\tau$ is the timing offset, $h(k)$ represents the $k$ th tap channel impulse response, $K$ is the number of taps in the channel, and $w(n)$ is a complex AWGN sample. After coarse frequency synchronization, the CFO-compensated received signal at the receiver is

$$
\begin{aligned}
\hat{r}(n) & =r(n) \cdot e^{\frac{-j 2 \pi(\epsilon+\Delta \epsilon) n}{N}} \\
& =e^{\frac{-j 2 \pi \Delta \epsilon n}{N}} \sum_{k=0}^{K-1} h(k) s(n-\tau-k)+\hat{w}(n),
\end{aligned}
$$

where $\Delta \epsilon$ denotes the residual $\mathrm{CFO}$ and $\hat{w}(n)=$ $w(n) e^{\frac{-j 2 \pi(\epsilon+\Delta \epsilon) n}{N}}$.

\subsection{Timing synchronization in the time-domain}

For any training-sequence-based communications system, timing synchronization can be easily achieved based on a well-designed timing metric in the time-domain. However, in this article, the proposed training sequence is a delta function with unit amplitude. Thus, if we perform the timing synchronization in the time-domain, the correlation function outputs $M(d)$ can be expressed as follows:

$$
\begin{aligned}
& \hat{\tau}=\arg \max _{d \in \Omega_{3}}\{|M(d)|\} \\
& M(d)=\sum_{n=0}^{N-1} \frac{\hat{r}(n+d) \cdot p(n)}{N}=\frac{\hat{r}(c+d)}{N},
\end{aligned}
$$

where $\hat{\tau}$ is the estimated timing offset, $|\hat{r}(n)|$ represents the absolute value of $\hat{r}(n), \Omega_{3}$ is the observation interval, 


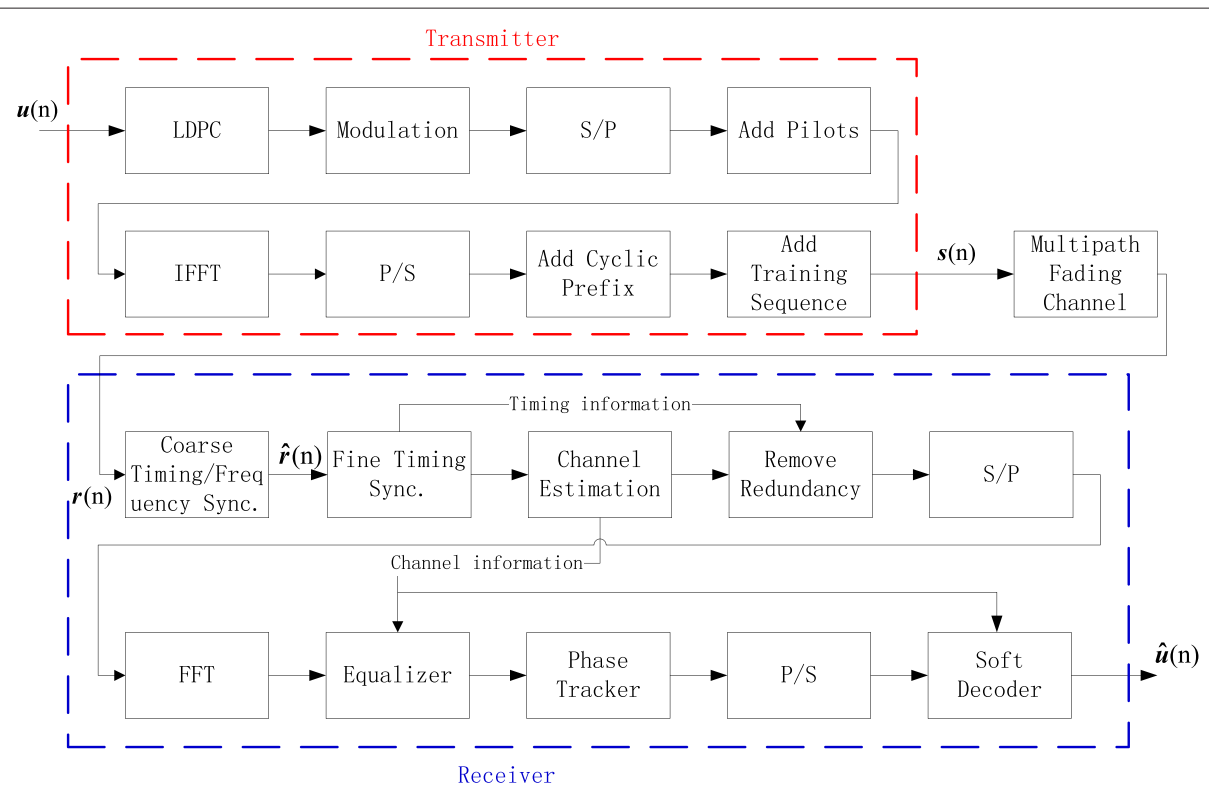

Figure 1 The training-sequence-based single-input single-output OFDM system architecture. Figure 1 represents the proposed training-sequence-based single-input single-output OFDM system.

$\Omega_{3}=\{0,1, \ldots, D-1\}$, and $D$ is the length of observation interval. If there is no residual CFO in Equation (2), we rewrite $|M(d)|$ in Equation (3) as follows:

$$
\begin{aligned}
& N \cdot|M(d)| \in \\
& \{|\hat{w}(c)|,|\hat{w}(c+1)|, \ldots,|\hat{w}(c+\tau-1)|, \\
& |h(0)+\hat{w}(c+\tau)|, \ldots,|h(K-1)+\hat{w}(c+\tau+K-1)|, \\
& |\hat{w}(c+\tau+K)|, \ldots,|\hat{w}(\tau+N-1)|, \\
& |h(0) x(0)+\hat{w}(N+\tau)|, \\
& \left.\left|\sum_{k=0}^{1} h(k) x(1-k)+\hat{w}(N+1+\tau)\right|, \ldots\right\} .
\end{aligned}
$$

From Equation (4), it is possible that all correlation function outputs related to the channel, $|h(k)+\hat{w}(c+\tau+k)|$, are smaller than the other elements in $N \cdot|M(d)|$ at low SNR, where $k \in\{0,1, \ldots, K-1\}$. Thus, we will have wrong timing estimates at low SNR as shown in Figure 2. In Figure 2, the delayed timing offset is 65 , and $c$ is 31 . Then, a maximum correlation function output near the 97th sample is expected. However, in Figure 2, a wrong timing estimate is obtained when SNR is $-5 \mathrm{~dB}$.

\section{The proposed approach}

\subsection{Coarse timing synchronization}

In order to achieve better timing synchronization performance at low SNR, from Section 2.2, a synchronization method in the time-domain is not suitable for the proposed preamble. However, much more information in the frequency-domain can be utilized to achieve better performance in timing synchronization. Consider there are two unit vectors, $p_{1}(n)=\delta\left(n-c_{1}\right)$ and $p_{2}(n)=\delta\left(n-c_{2}\right)$, the cross-correlation function outputs between these two unit vectors in the frequency-domain are

$$
\frac{1}{N} \sum_{m=0}^{N-1}\left\{e^{\frac{-j 2 \pi m c_{1}}{N}} \times e^{\frac{j 2 \pi m c_{2}}{N}}\right\}=\left\{\begin{array}{l}
0, \forall c_{1} \neq c_{2} \\
1, c_{1}=c_{2}
\end{array}\right.
$$

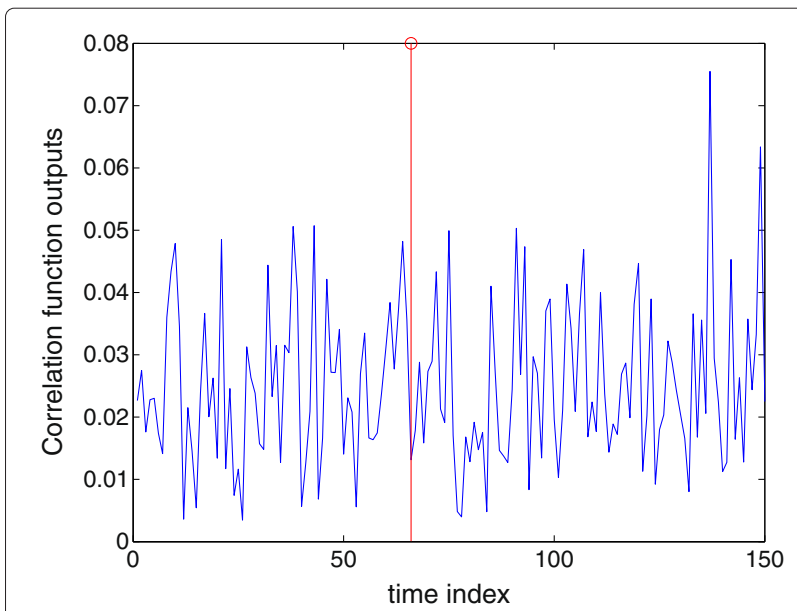

Figure 2 The correlation function output based on Equation (3). Figure 2 represents the correlation function outputs based on Equation (3), where $\mathrm{SNR}=-5 \mathrm{~dB}$, timing offset $(\tau)$ is $65, N=64$, $N_{C P}=16, \ell=17, C=31, K=6$, and the red-line indicates the correct delayed timing in the channel. 
where $\forall c_{1}, c_{2} \in\{0,1, \ldots, N-1\}$ and $m$ represents the subcarrier index. Therefore, based on Equation (5), a frequency-domain timing synchronization scheme based on the cross-correlation function outputs is proposed. By employing the cross-correlation function in the frequency-domain, a timing metric for coarse timing estimation $\left(\tau_{c}\right)$ is given by

$$
\begin{aligned}
& \tau_{c}=\arg \max _{d_{1} \in \Omega_{c}}\left\{M_{1}\left(d_{1}\right)\right\} \\
& M_{1}\left(d_{1}\right)=\left|\Re\left\{U\left(d_{1}\right)\right\}\right|+\left|\Im\left\{U\left(d_{1}\right)\right\}\right| \\
& U\left(d_{1}\right)=\frac{1}{N} \sum_{m=0}^{N-1}\left\{R\left(d_{1}, m\right) \times b^{*}(m)\right\} \\
& R\left(d_{1}, m\right)=\sum_{n=0}^{N-1} \hat{r}\left(n+d_{1}\right) \cdot e^{\frac{-j 2 \pi m n}{N}},
\end{aligned}
$$

where $\Re\{u\}$ and $\mathfrak{s}\{u\}$ represent the real part and the imaginary part of $u$, respectively, $R(\cdot, \cdot)$ denotes the Fourier transform of the received signal $\hat{r}, b^{*}(m)$ is the complex conjugate of $b(m),|b(m)|$ denotes the absolute value of $b(m), b(m)=e^{\frac{-j 2 \pi m c}{N}}, \Omega_{c}$ is the observation interval, $\Omega_{c}=$ $\{0,1, \ldots, L-1\}, d_{1}$ is the time index, $d_{1} \in\{0,1, \ldots, L-$ $1\}, R\left(d_{1}, m\right)$ represents the value of the $m$ th subcarrier with respect to $d_{1}, U\left(d_{1}\right)$ is the cross-correlation function output in the frequency-domain, and $L$ is the length of observation interval. In addition, if there is no CFO in Equation (1), $M_{1}\left(d_{1}\right)$ in Equation (6) can be further modified to

$$
M_{1}\left(d_{1}\right)=\left|\Re\left\{U\left(d_{1}\right)\right\}\right| .
$$

However, by using both real part and imaginary part of the cross-correlation function output, more information can be utilized to obtain a better coarse timing estimate.

Assume an unit vector $p_{i}(n)=\delta\left(n-c_{i}\right)$ is transmitted over a two-ray multipath fading channel $\left(\mathbf{h}_{i}\right)$ without AWGN, a time delay is given by $\tau$, and the power profile of the channel is equal to $\{0.3,0.7\}$, where $c_{i} \in\{0,1, \ldots, N-$ $1\}$. Therefore, the received signal is

$$
r_{i}(n)=\sum_{k=0}^{1} h_{i}(k) \delta\left(n-c_{i}-k-\tau\right) .
$$

Consider $\mathbf{h}_{i}^{T}$ is equal to $[0.3873+0.3873 j 0.5916+$ $0.5916 j]$. Then, the received signal $r_{i}(n)$ is

$$
r_{i}(n)=\left\{\begin{array}{l}
0.3873+0.3873 j, n=c_{i}+\tau \\
0.5916+0.5916 j, n=c_{i}+\tau+1 \\
0, \text { else }
\end{array}\right.
$$

Based on Equation (6), the cross-correlation function output $\left(M_{1}\left(d_{1}\right)\right)$ is

$$
M_{1}\left(d_{1}\right)=\left\{\begin{array}{l}
0.7746, d_{1}=\tau \\
1.1836, d_{1}=\tau+1 \\
0, \text { else. }
\end{array}\right.
$$

Thus, a coarse timing estimate $\left(\tau_{c}\right)$ is

$$
\tau_{c}=\tau+1 \text {. }
$$

From Equation (10), although $M_{1}\left(d_{1}\right)$ gives a maximum value when $d_{1}$ is at the delayed timing of the path with the largest gain in multipath fading channels, the actual delayed timing cannot be obtained.

In addition, for the general channel impulse response $\mathbf{h}$ in Equation (1), the received training sequence is

$$
\begin{aligned}
\hat{\mathbf{r}}= & {\left[\begin{array}{lll}
0 & \ldots 0 e^{\frac{-j 2 \pi \Delta \epsilon\left(\tau+c_{i}\right)}{N}} h(0) \delta\left(\tau+c_{i}\right) \\
& e^{\frac{-j 2 \pi \Delta \epsilon\left(\tau+c_{i}+1\right)}{N}} h(1) \delta\left(\tau+c_{i}+1\right) \ldots \\
& e^{\frac{-j 2 \pi \Delta \epsilon\left(\tau+c_{i}+K-1\right)}{N}} h(K-1) \delta\left(\tau+c_{i}+K-1\right) 0 \ldots 0
\end{array}\right]^{T} } \\
& +\hat{\mathbf{w}}
\end{aligned}
$$

where $E[\hat{\mathbf{w}}]=0$ and $E[\cdot]$ is the expectation operation. Then, the corresponding timing metric $M_{1}\left(d_{1}\right)$ is

$$
\begin{aligned}
M_{1}\left(d_{1}\right)= & \left\{\begin{array}{l}
|h(k)| \cdot\left\{\left|\cos \left(\frac{2 \pi \Delta \epsilon\left(\tau+c_{i}+k\right)}{N}-\theta_{k}\right)\right|+\right. \\
\left.\left|\sin \left(\frac{2 \pi \Delta \epsilon\left(\tau+c_{i}+k\right)}{N}-\theta_{k}\right)\right|\right\}, d_{1} \in\{\tau+k\} \\
0, \text { else }
\end{array}\right. \\
= & \left\{\begin{array}{l}
|h(k)| \cdot A_{k}, d_{1} \in\{\tau+k\} \\
0, \text { else, }
\end{array}\right.
\end{aligned}
$$

where $k \in\{0, \ldots, K-1\}, h(k)=|h(k)| e^{j \theta_{k}},|h(k)|=$ $\sqrt{(\Re\{h(k)\})^{2}+(\Im\{h(k)\})^{2}}$, and $\theta_{k}=\tan ^{-1} \frac{\Im\{h(k)\}}{\Re\{h(k)\}}$. From Equation (13), we can easily obtain

$$
\sqrt{2} \geq A_{k} \geq 1
$$

and

$$
\sqrt{2} \cdot|h(k)| \geq M_{1}(\tau+k) \geq|h(k)| .
$$

\subsection{Fine time adjustment}

Let us pay attention to Equations (10) and (13). In Equations (10) and (13), two cross-correlation function outputs related to the multipath fading channel have a strong connection. The correct timing offset can be found using a simple threshold on cross-correlation outputs. Then, by utilizing the cross-correlation outputs at two adjacent timing indices, we can obtain the actual delayed 
timing in the channels. First, a sliding observation vector $(\mathrm{SOV}) \mathbf{v}$ based on the coarse timing estimate is utilized to perform the fine time adjustment, where

$$
\begin{aligned}
\mathbf{v}^{T} & =\left[\tau_{c} \tau_{c}-1 \tau_{c}-2 \ldots \tau_{c}-V+1\right] \\
& =\left\{v(n), \forall n \in \Omega_{v}\right\},
\end{aligned}
$$

the length of the SOV is $V$, and $\Omega_{v}=\{0,1, \ldots, V-1\}$. If $M_{1}(v(i+1))>\beta \cdot M_{1}(v(i))$ and $M_{1}(v(i+2))<\beta \cdot M_{1}(v(i+$ $1)$ ), the final timing estimate $(\hat{\tau})$ is $v(i+1)$, where $\beta$ is a threshold and $i \in \Omega_{v}$. The detailed procedure of fine time adjustment is described in Algorithm 1.

\section{Algorithm 1. Fine time adjustment. \\ Initial Inputs: $M_{1}(v(i)), \mathbf{v}$}

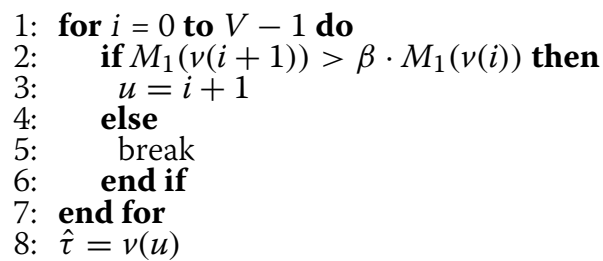

In Algorithm $1, \beta$ is utilized to perform the fine time adjustment. Based on Equation (13), $\tau_{c}$ is approximately equal to the timing index of the path with the largest gain in multipath fading channels. Therefore, the time difference between the timing index of the path with the largest gain and the timing index of the first delayed path in the channels is approximately equal to $N_{I}-1$, where the actual number of iterations executed in Algorithm 1 is $N_{I}$ and $N_{I}<V$. Assume the second path has the largest power in the channel. If the correct delayed timing is obtained, $M_{1}(\tau)>\beta \cdot M_{1}(\tau+1)$ and $M_{1}(\tau-1)<\beta \cdot M_{1}(\tau)$ must be satisfied. Based on these two conditions, we have

$$
0<\beta<\frac{M_{1}(\tau)}{M_{1}(\tau+1)}=\frac{|h(0)| \cdot A_{0}}{|h(1)| \cdot A_{1}}=\sqrt{\frac{|h(0)|^{2}}{|h(1)|^{2}}} \cdot \frac{A_{0}}{A_{1}} .
$$

Because the bound of $\frac{A_{0}}{A_{1}}$ is

$$
\frac{1}{\sqrt{2}} \leq \frac{A_{0}}{A_{1}} \leq \sqrt{2}
$$

we obtain the bound of the threshold $\beta$ given by

$$
0<\beta<\sqrt{\frac{|h(0)|^{2}}{|h(1)|^{2}}} \cdot \frac{1}{\sqrt{2}} .
$$

In general, consider the $k$ th tap has the largest power in the channel. Then, the threshold $\beta$ in the fine time adjustment can be chosen by satisfying:

$$
0<\beta<\sqrt{\frac{|h(k-1)|^{2}}{|h(k)|^{2}}} \cdot \frac{1}{\sqrt{2}}
$$

where $k>0$. Moreover, if the first path is the path with the largest gain in the channel, the threshold can be easily set to

$$
0<\beta<\sqrt{\frac{\left|h\left(k^{\prime}\right)\right|^{2}}{|h(0)|^{2}}} \cdot \frac{1}{\sqrt{2}},
$$

where the $k^{\prime}$ th tap has the second-largest power in the channel.

\subsection{Channel estimation}

After the final timing estimate $(\hat{\tau})$ is found, the channel response in the frequency-domain could be obtained in a simple way. Therefore, the estimated channel response in the frequency-domain is

$$
\hat{H}(m)=\frac{\sum_{n=0}^{N-1} \hat{r}(n+\hat{\tau}) \cdot e^{\frac{-j 2 \pi m n}{N}}}{b(m)},
$$

where $\hat{H}(m)$ is the estimated channel response on the $m$ th subcarrier.

\section{Simulation results}

A packet-based low-density parity-check (LDPC) coded single-input single-output (SISO) OFDM system was used for simulations, where each codeword is encoded with code $(1600,800)$ [17] and each packet consists of a training sequence followed by 17 random OFDM data symbols. The structure of OFDM data symbols follows the IEEE 802.11a standard defined in [9], where $N=64$ and $N_{C P}=16$. The training sequence of each packet is an unit vector with unit amplitude in the time-domain, where $c=31$ and the power of the training sequence is 1/64. Quaternary phase-shift keying modulation was adopted in simulations. For each packet transmission, the residual CFO was modeled as a random variable that is uniformly distributed within \pm 0.1 OFDM subcarrier spacing. In addition, the phase tracker based on the pilots in the frequency-domain is utilized to compensate the phase error [9].

We evaluate the proposed approach and other related schemes $[7,9,15]$ under 6-path Rayleigh channels, where the power profiles of their first four taps are described in Table 1 and $\sigma_{i}^{2}$ represents the $i+1$ th tap power in the channel. A delayed timing offset $(\tau)$ is given by 65 samples. Channel Models I and II (CH I and $\mathrm{CH}$ II) represent multipath fading channels with NLOS propagation, and

Table 1 The power profiles of different channel models

\begin{tabular}{cccc}
\hline & CH I (NLOS) & CH II (NLOS) & CH III (LOS) \\
\hline$\sigma_{0}^{2}$ & 0.3432 & 0.1885 & 0.5211 \\
$\sigma_{1}^{2}$ & 0.6211 & 0.3223 & 0.4338 \\
$\sigma_{2}^{2}$ & 0.0329 & 0.48 & 0.0420 \\
$\sigma_{3}^{2}$ & 0.0015 & 0.0079 & 0.0023 \\
\hline
\end{tabular}


Channel Model III (CH III) is a typical multipath fading channel with LOS propagation. For $\mathrm{CH} \mathrm{I}$, the power of second tap dominates all channel taps. As for $\mathrm{CH}$ II, the third tap has the strongest power in the channel, and it is the worst channel model to evaluate the performance of timing synchronization in this article. Moreover, assume all channels are quasi-stationary during each packet transmission.

The main motivation of this article is to achieve perfect timing synchronization in very low SNR environments by using unit vectors in the $N$ dimensional Cartesian coordinate system. In Algorithm 1, although the number of iterations is defined by $V$, the number of iterations actually depends on the comparison between cross-correlation function outputs. In this article, the actual number of iterations executed in Algorithm $1\left(N_{I}\right)$ is less than 3. In addition, in $\mathrm{CH}$ I and $\mathrm{CH}$ II, the thresholds $\beta$ in the fine time adjustment should be less than 0.5256 and 0.5794 , respectively. Therefore, based on the SNR, we employ different thresholds $\beta$ defined in fine time adjustment to achieve better performance. For SNR $\leq 0 \mathrm{~dB}, \beta$ is 0.5 . As for SNR $>0 \mathrm{~dB}, \beta$ is 0.3 . In [7], the time-domain training sequence is generated by a Golay complementary sequence, i.e., \pm 1 , and the length of the time-domain training sequence is $N$. In addition, the actual threshold in [7] is $\eta\left|\hat{h}_{\max }\right|$, where $\eta$ is a threshold factor and $\left|\hat{h}_{\max }\right|$ is the strongest channel tap gain estimate. The same criterion for $\beta$ is applied to $\eta$. Moreover, in this article, pre-simulations and mathematical derivations are not required to choose the threshold for fine time adjustment $[7,8,11]$. Let $L=200$ and $V=$ 50. Therefore, the length of the interval for fine-timing estimation in [7] is also set to 50. For [15], we use four concatenated cyclic prefixes to perform the timing synchronization. The corresponding results are reported in Figure 3 . The perfect timing synchronization is defined as the successful acquisition of the position of the first tap in channel models.

In Figure 3, simulation results show that our proposed approach has better performance in timing synchronization at very low SNR. In CH I, the proposed approach achieves perfect timing synchronization when SNR exceeds $1 \mathrm{~dB}$. As for $\mathrm{CH}$ II, perfect timing synchronization is achievable using the proposed algorithm when $\mathrm{SNR}=6 \mathrm{~dB}$. In $\mathrm{CH}$ III, the proposed approach achieves perfect timing synchronization when SNR exceeds $-5 \mathrm{~dB}$. Moreover, for $c=63$, the perfect timing synchronization is achievable at low SNR by only appending one sample to the front of the transmitted packet. The synchronization methods used in IEEE 802.11 standards lead to the delayed path with the maximum gain in channels. Also, the standardized methods are only suitable for the channels with LOS propagation. In CH III, the first tap power is approximately equal to the second tap power. Thus, higher SNR is needed to achieve perfect timing synchronization

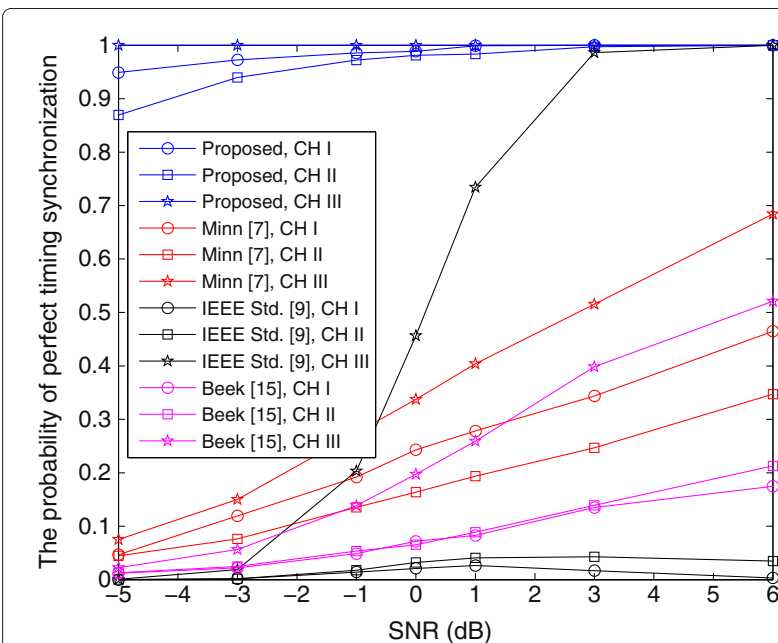

Figure 3 The Probability of perfect timing synchronization. Figure 5 represents the probability of perfect timing synchronization, $\operatorname{prob}(\tau=\hat{\tau})$, where $\operatorname{prob}(\cdot)$ is the probability function and $\hat{\tau}$ is the estimated timing offset.

for [9]. As for [7], the reason why the scheme has poor performance of perfect timing synchronization is that AWGN affects the entire fine time adjustment process at low SNR, especially in $\mathrm{CH}$ I and $\mathrm{CH}$ II. Therefore, low SNR and wide interval for fine time adjustment significantly degrade the performance in $[7,8,11]$. For [15], the proposed timing estimator is only suitable in AWGN channel at high SNR. Thus, the time estimator of [15] has poor performance in all channel models. Besides the probability of perfect timing synchronization, we also evaluate the bias and root mean squared error (RMSE) of each approach. The simulation results are listed in Figures 4 and 5. In Figures 4 and 5, our proposed approach has

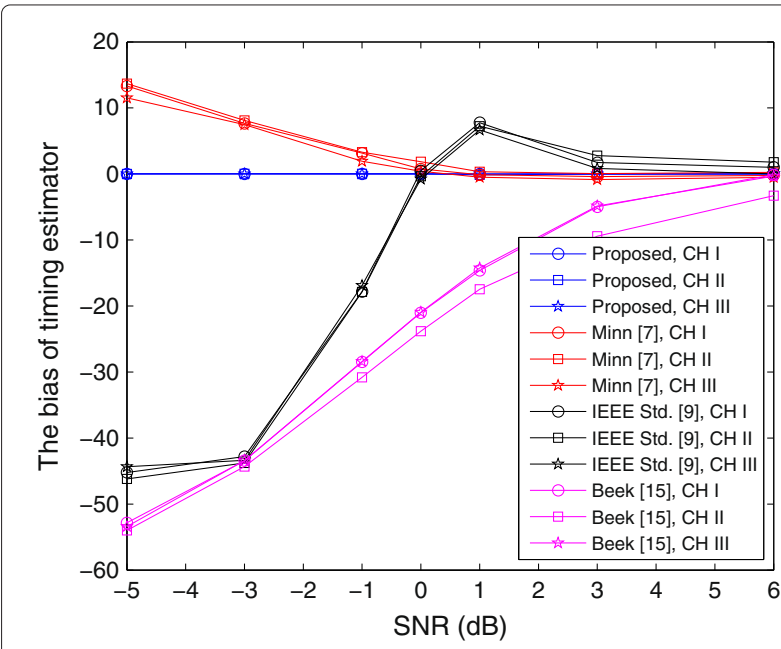

Figure 4 The Bias of timing estimator. Figure 6 represents the bias of timing estimator, $E[\hat{\tau}-\tau]$, where $E[\cdot]$ is the expectation function. 


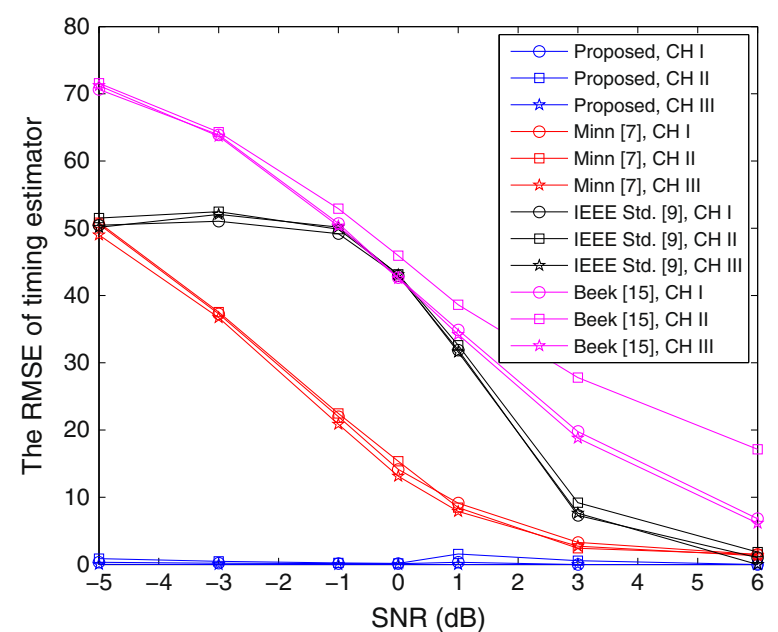

Figure 5 The root mean squared error of timing estimator.

Figure 7 represents the root mean squared error of timing estimator, $\sqrt{E\left[|\hat{\tau}-\tau|^{2}\right]}$.

better performance than other methods in $[7,9,15]$. In Figure 4, our proposed approach performs almost unbiased at any low SNR, and wide interval for fine time adjustment in [7] leads the time estimator to have negative biases. In Figure 5, the proposed approach achieves zero RMSE due to the ability to identify the first arrival path in channels.

In Figure 6, we compare the proposed approach with [7] in terms of bit-error-rate (BER). For CH III, zero BER is achievable for the system using our proposed approach when SNR exceeds $6 \mathrm{~dB}$, because there is no timing error to process the received signals. As for $\mathrm{CH} \mathrm{I}$ and $\mathrm{CH}$ II, zero BER is still achievable when SNR exceeds $8 \mathrm{~dB}$. In addition, the BER performance of [7] decreases slowly and still does not reach $10^{-2}$ when $\mathrm{SNR}=8 \mathrm{~dB}$ in $\mathrm{CH}$ I and $\mathrm{CH}$

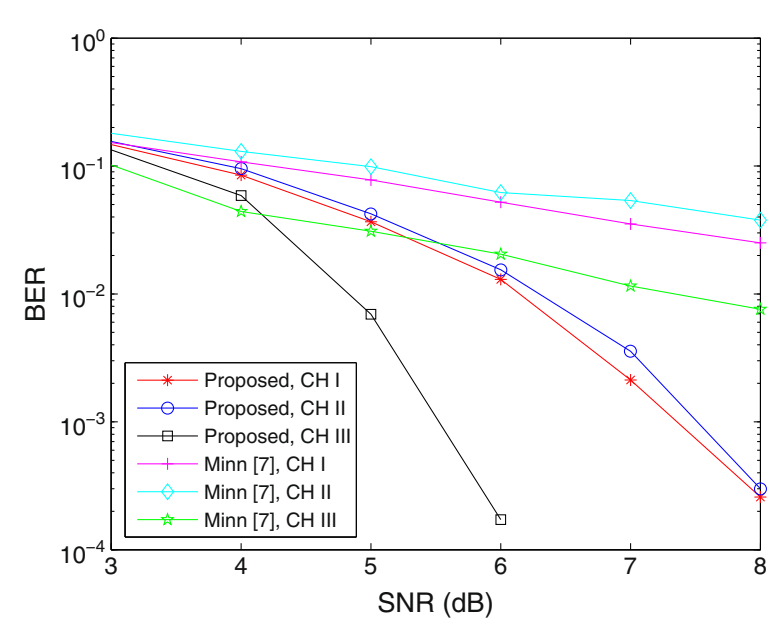

Figure 6 BER comparisons with [7] in all channel models.

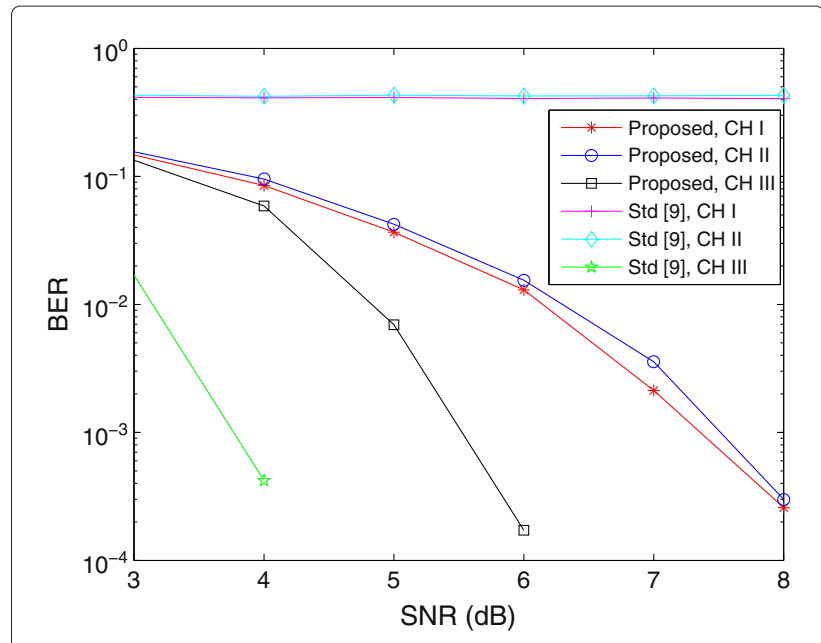

Figure 7 BER comparisons with [9] in all channel models.

II. As for Figure 6, we apply the synchronization methods in [9] to find the timing offset, and then utilize the perfect channel information to compensate the received signal. In Figure 7, zero BER is achievable when SNR exceeds $4 \mathrm{~dB}$ in $\mathrm{CH}$ III, because perfect timing synchronization is achieved. For $\mathrm{CH}$ I and $\mathrm{CH}$ II, there are two error floors shown in Figure 7, because timing errors degrade the system performance by introducing ISI and ICI. In addition, from Figure 7, the channel estimation in our proposed algorithm has a $2 \mathrm{~dB}$ loss compared to the Cramér-Rao lower bound.

\section{Conclusion}

In this article, we have developed a semiblind timing synchronization and channel estimation scheme for OFDM systems based on unit vectors. We also developed a fine time adjustment algorithm to find the actual position of the first arrival path in LOS and NLOS channel models. Based on a simple threshold without any pre-simulation and theoretical derivation, the proposed fine time adjustment algorithm outperforms conventional schemes even at very low SNR. Simulation results show that there are no timing errors in our proposed time estimator when SNR exceeds $6 \mathrm{~dB}$. In addition, zero BER is also achievable for a LDPC coded SISO OFDM system when SNR exceeds $8 \mathrm{~dB}$.

\section{Competing interests}

The authors declare that they have no competing interests.

Received: 12 October 2012 Accepted: 4 December 2012

Published: 2 January 2013

\section{References}

1. H Sari, G Karam, I Jeanclaude, Transmission techniques for digital terrestrial TV broadcasting, IEEE Commun. Mag. 33, 100-109 (1995)

2. U Reimers, Digitalvideo broadcasting, IEEE Commun. Mag. 36, 104-110 (1998) 
3. L Cimini, J Chuang, N Sollenberger, Advanced internet services, IEEE Commun. Mag. 36, 150-159 (1998)

4. J Montojo, L Milstein, Channel estimation for non-ideal OFDM systems, IEEE Trans. Commun. 58(1), 146-156 (2010)

5. T Schmidl, D Cox, Robust frequency and timing synchronization for OFDM, IEEE Trans. Commun. 45(12), 1613-1621 (1997)

6. H Minn, M Zeng, $\vee$ Bhargava, On timing offset estimation for OFDM systems, IEEE Commun. Lett. 4(7), 242-244 (2000)

7. H Minn, $V$ Bhargava, K Letaief, A robust timing and frequency synchronization for OFDM systems, IEEE Trans. Wirel. Commun. 2(4), 822-839 (2003)

8. H Minn, V Bhargava, K Letaief, A combined timing and frequency synchronization and channel estimation for OFDM, IEEE Trans. Commun. 54(3), 416-422 (2006)

9. IEEE std 802.11, Wireless LAN Medium Access Control (MAC) and Physical Layer (PHY) specifications (2007)

10. Y Mostofi, D Cox, A robust timing synchronization design in OFDM systems-part II: high-mobility cases, IEEE Trans. Wirel. Commun. 6(12), 4340-4348 (2007)

11. C-L Wang, H-C Wang, On joint fine time adjustment and channel estimation for OFDM systems, IEEE Trans. Wirel. Commun. 8(10), 4940-4944 (2009)

12. H Abdzadeh-Ziabari, M Shayesteh, Robust timing and frequency synchronization for OFDM systems, IEEE Trans. Veh. Technol. 60(8), 3646-3656 (2011)

13. T-L Kung, K Parhi, in IEEE Conference on Electro/Information Technology. Frequency domain symbol synchronization for OFDM systems, (Minnesota, USA, 2011), pp. 1-5

14. T-L Kung, K Parhi, Optimized joint timing synchronization and channel estimation for OFDM systems, IEEE Wirel. Commun. Lett. 1(3), 149-152 (2012)

15. J Beek, M Sandell, P Börjesson, ML estimation of time and frequency offset in OFDM systems, IEEE Trans. Signal Process. 45(7), 1800-1805 (1997)

16. S Ma, X Pan, GH Yang, TS Ng, Blind symbol synchronization based on cyclic prefix for OFDM systems, IEEE Trans. Veh. Technol. 58(4), 1746-1751 (2009)

17. R Neal. http://www.cs.utoronto.ca/ radford/ldpc.software.html

doi:10.1186/1687-6180-2013-1

Cite this article as: Kung and Parhi: Semiblind frequency-domain timing synchronization and channel estimation for OFDM systems. EURASIP Journal on Advances in Signal Processing 2013 2013:1.

\section{Submit your manuscript to a SpringerOpen ${ }^{\mathcal{O}}$ journal and benefit from:}

- Convenient online submission

- Rigorous peer review

- Immediate publication on acceptance

- Open access: articles freely available online

- High visibility within the field

- Retaining the copyright to your article

Submit your next manuscript at $\boldsymbol{\wedge}$ springeropen.com 